\title{
POTENTIAL, ELECTRIC FIELD AND SURFACE CHARGES CLOSE TO THE BATTERY FOR A RESISTIVE CYLINDRICAL SHELL CARRYING A STEADY LONGITUDINAL CURRENT
}

\author{
$\begin{array}{lll}\text { J. A. Hernandes } & \text { E. Capelas de Oliveira } & \\ & \text { A. K. T. } \text { Assis }^{3}\end{array}$ \\ Recibido el 12 de mayo de 2004, aceptado el 30 de agosto de 2004
}

\begin{abstract}
RESUMEN
En este trabajo consideramos una capa resistiva cilíndrica que transporta una corriente constante. Una batería genera la corriente en el centro del conductor. Estudiamos el comportamiento del potencial, campo eléctrico y cargas superficiales cerca de la batería.
\end{abstract}

Palabras clave: Potencial eléctrico, potencial cerca de la batería, cargas superficiales.

ABSTRACT

In this work we consider a long, resistive cylindrical shell carrying a steady current. A battery in the middle of the wire generates the current. We study the behavior of the potential, electric field and surface charges close to the battery.

Keywords: Electric potential, potential close to the battery, surface charges.

\section{INTRODUCTION}

There has been recently a great interest in the electric field outside resistive conductors carrying steady currents and a number of problems have been published in the literature: coaxial cables, [14], [8], [13], [1] and [2]; cylindrical shell with azimuthal current, [12], [10] and [16]; planes, [13]; twin leads, [15] and [4]; a long strip, [9]; and a long straight cylindrical conductor with longitudinal current, [5]. In this last case it has only been considered the region far from the battery. It was then found that the potential and surface charges vary linearly with the longitudinal component. Here we analyse the situation close to the battery in order to understand the behaviour of surface charges at a discontinuity in the potential.

We consider a hollow cylindrical shell of radius $a$ and length $\ell$ with $\ell>>a$. The shell has an uniform resistivity and carries a steady current $I$ along the positive $z$ direction which coincides with its axis. We suppose an idealized linear battery at $(\rho, \varphi, z)=(a, \varphi, 0)$, see Fig. 1, in analogy with Heald' $\mathrm{s}$ treatment which considered a "line" battery at $(\rho, \varphi, z)=(a, \pi, z)$ driving current azimuthally in a uniform cylindrical resistive sheet, [10]. We suppose a vacuum inside and outside the shell.

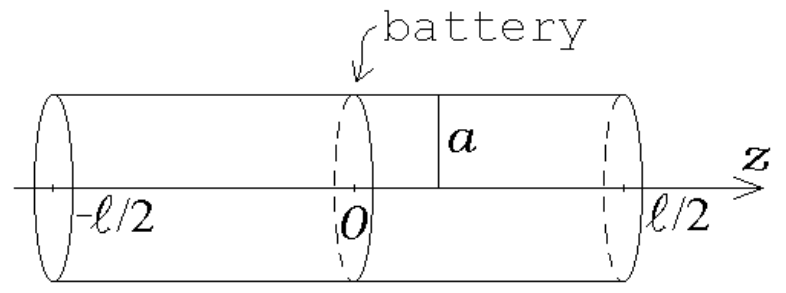

Fig. 1 A resistive cylindrical shell of radius $a$ and length $\ell$ carrying a steady current in the $z$ direction. There is a linear battery in $z=0$ generating a difference of potential of $2 \phi_{0}$.

Ohm' s law can be written as $\vec{J}=g \vec{E}$, where $\vec{J}=(I / 2 \pi a t) \hat{z} \quad$ is the volume current density ( $t<<a$ being the thickness of the shell), $g$ the conductivity of the wire and $\vec{E}$ the driving electric field. We are supposing $g$ and $\vec{J}$ as constants independent of $\varphi, z$ and time. Therefore the same

\footnotetext{
1 Instituto de Física 'Gleb Wataghin', Universidade Estadual de Campinas - Unicamp, 13083-970 Campinas, São Paulo, Brasil, julioher@ifi.unicamp.br

${ }_{2}^{2}$ Departamento de Matemática Aplicada, Imecc - Unicamp, 13081-970 Campinas (SP) Brasil, capelas@ime.unicamp.br

${ }^{3}$ Instituto de Física ‘Gleb Wataghin', Universidade Estadual de Campinas - Unicamp, 13083-970 Campinas, São Paulo, Brasil, assis@ifi.unicamp.br; homepage: http://www.ifi.unicamp.br/ assis/
} 
must be true for $\vec{E}$. As $\vec{E}=-\nabla \phi, \phi$ being the potential, this implies that $\phi$ along the shell $(\rho=a)$ must be a linear function of $z$. Supposing that the battery generates a potential difference of $2 \phi_{0}$ between its two terminals located at $z \rightarrow 0^{-}$and $z \rightarrow 0^{+}$, the linear potential at $\rho=a$ can be written as (Fig. 2):

$$
\begin{aligned}
& \phi(a, \varphi, z<0)=\left(\phi_{R}-\phi_{L}\right) \frac{z}{\ell}+\frac{\phi_{R}+\phi_{L}}{2}-\phi_{0} \\
& \phi(a, \varphi, z>0)=\left(\phi_{R}-\phi_{L}\right) \frac{z}{\ell}+\frac{\phi_{R}+\phi_{L}}{2}+\phi_{0}
\end{aligned}
$$

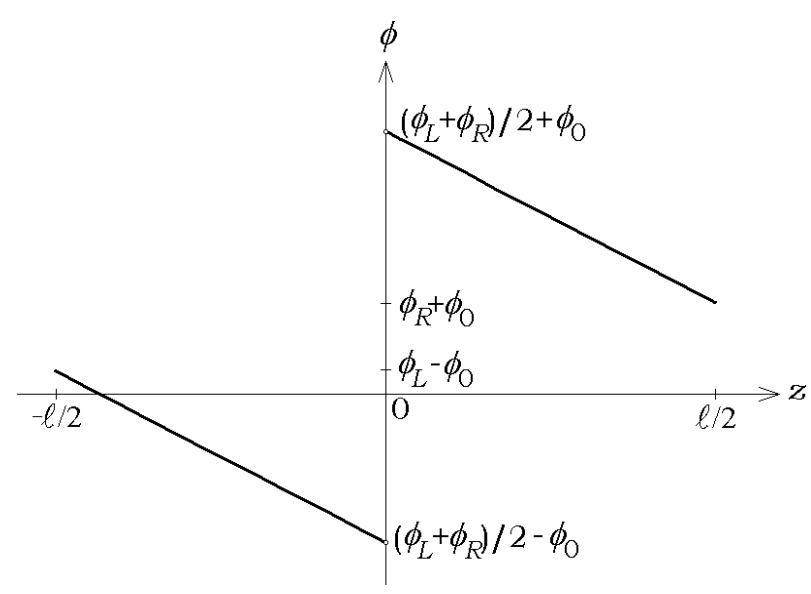

Fig. 2 Potential along the shell.

Our goal is to find the potential and electric field at $\rho<a$ and at $\rho>a$ given the boundary conditions above. Then we can obtain the surface charge densities at the inner and outer surfaces of the shell.

This problem can be separated in two parts: a) the electrostatic situation of a cylindrical shell separated at $z=0$ by a thin insulating barrier held at $-\phi_{0}$ for $z<0$ and at $\phi_{0}$ for $z>0$; and b) a continuous linear potential $\phi(a, \varphi, z)=\left(\phi_{R}-\phi_{L}\right) z / \ell+\left(\phi_{R}+\phi_{L}\right) / 2 \quad$ for $-\ell / 2 \leq z \leq \ell / 2$, see Fig. 3. The first part has been partially solved in [7] and the second one in [5]. Our intention is to deepen these studies in order to understand the behaviour close to the battery.

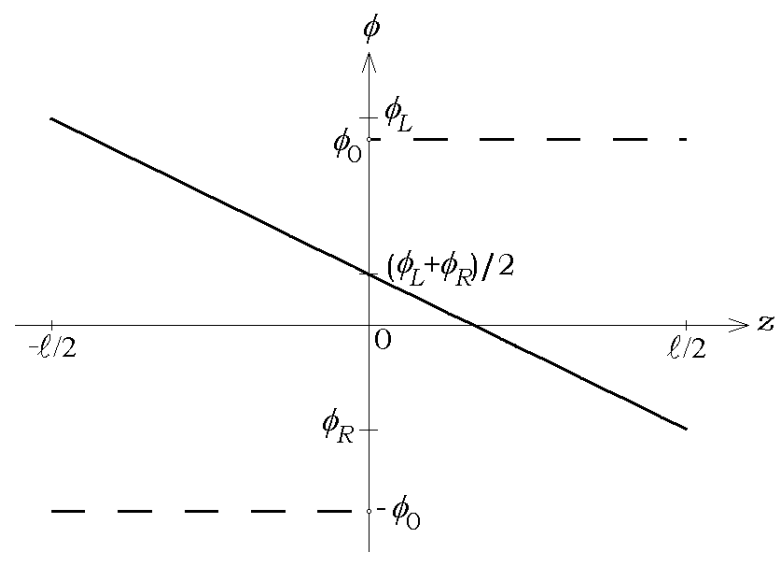

Fig. 3 The potential of Fig. 2 can be decomposed in two parts: the shell held at constant but discontinuous potentials (dashed lines), and the shell with a continuous potential varying linearly with the longitudinal coordinate (continuous line).

\section{ELECTROSTATIC SOLUTION OF THE CYLINDRICAL SHELL WITH IS TWO HALVES HELD AT CONSTANT AND OPPOSITE POTENTIALS}

Suppose two semi-infinite cylindrical shells, located at $z<0$ and $z>0$, see Fig. 1. The shell at $z<0$ is held at the constant potential $-\phi_{0}$, while the shell at $z>0$ is held at $\phi_{0},[17]$. We can solve Laplace' s equation, $\nabla^{2} \phi=0$, in cylindrical coordinates using separation of variables in the form $\phi_{j k}(\rho, \varphi, z)=R_{j k}(\rho) \Phi_{j}(\varphi) Z_{k}(z)$, where the functions $R_{j k}, \Phi_{j}$ and $Z_{k}$ obey the equations:

$$
\begin{gathered}
\frac{d^{2} R_{j k}}{d \rho^{2}}+\frac{1}{\rho} \frac{d R_{j k}}{d \rho}-\left(k^{2}+\frac{j^{2}}{\rho^{2}}\right) R_{j k}=0, \\
\frac{d^{2} \Phi_{j}}{d \varphi^{2}}-j^{2} \Phi_{j}=0, \\
\frac{d^{2} Z_{k}}{d z^{2}}+k^{2} Z_{k}=0
\end{gathered}
$$

The final solution $\phi(\rho, \varphi, z)$ is a linear combination of all possible solutions $\phi_{j k}(\rho, \varphi, z)$.

Due to the rotational symmetry of the system, the solution will not depend on $\varphi$. This means $j=0$ and $\Phi_{j}=$ constant . 
In this situation, the boundary conditions are antisymmetric about the $z$ coordinate, $\phi(a, \varphi,-z)=-\phi(a, \varphi, z)$, so the solution must also be anti-symmetric at all points: $\phi(\rho, \varphi,-z)=-\phi(\rho, \varphi, z)$ and $\phi(\rho, \varphi, 0)=0$. Additionally, we must have a limited solution in both $\rho$ and $z$ coordinates, $|\phi(\rho, \varphi,|z| \rightarrow \infty)| \leq \phi_{0}<\infty \quad$ and $\quad|\phi(\rho \rightarrow \infty, \varphi, z)| \rightarrow 0$. This leads to a solution for $Z_{k}$ of the form: $Z_{k}(z)=\sin (k z)$. The equation for $R_{j k}$ is the modified Bessel equation, whose solutions are $I_{j}(k \rho)$ (regular for $\rho \rightarrow 0$ and irregular for $\rho \rightarrow \infty)$ and $K_{j}(k \rho)$ (regular for $\rho \rightarrow \infty$ and irregular for $\rho \rightarrow 0$ ), [6].

The general solution has then the form:

$$
\begin{aligned}
& \phi(\rho \leq a, \varphi, z)=\int_{0}^{\infty} A_{k} I_{0}(k \rho) \sin (k z) d k \\
& \phi(\rho \geq a, \varphi, z)=\int_{0}^{\infty} B_{k} K_{0}(k \rho) \sin (k z) d k
\end{aligned}
$$

where the coefficients $A_{k}$ and $B_{k}$ must be determined from the boundary conditions. Eqs. (4) and (5) can be seen as a sine Fourier transform of a function $\Psi$ :

$$
\begin{aligned}
& \Psi(\rho, \varphi, k)=\sqrt{\frac{2}{\pi}} \int_{0}^{\infty} \phi(\rho, \varphi, z) \sin (k z) d z= \\
& = \begin{cases}\sqrt{\pi / 2} A_{k} I_{0}(k \rho), & \rho \leq a \\
\sqrt{\pi / 2} B_{k} K_{0}(k \rho) & \rho \geq a\end{cases}
\end{aligned}
$$

Calculating Eq. (6) in $\rho=a$ and applying the boundary conditions $\phi(a, \varphi, z<0)=-\phi_{0}$ and $\phi(a, \varphi, z>0)=\phi_{0}$ yields the coefficients $A_{k}$ and $B_{k}$ :

$$
A_{k}=\frac{2 \phi_{0}}{\pi k I_{0}(k a)}, \quad B_{k}=\frac{2 \phi_{0}}{\pi k K_{0}(k a)}
$$

The final solution can be written as (see Appendix):

$$
\begin{aligned}
& \phi(\rho \leq a, \varphi, z)=\frac{2 \phi_{0}}{\pi} \int_{0}^{\infty} \frac{I_{0}(k \rho)}{I_{0}(k a)} \frac{\sin (k z)}{k} d k \\
& =2 \phi_{0} \frac{z}{|z|}\left[\frac{1}{2}-\sum_{n=1}^{\infty} \frac{J_{0}\left(x_{n} \rho / a\right)}{J_{1}\left(x_{n}\right)} \frac{\mathrm{e}^{-x_{n}|z| / a}}{x_{n}}\right] \\
& \phi(\rho \geq a, \varphi, z)=\frac{2 \phi_{0}}{\pi} \int_{0}^{\infty} \frac{K_{0}(k \rho)}{K_{0}(k a)} \frac{\sin (k z)}{k} d k
\end{aligned}
$$

In Eq. (9), $x_{n}$ are the zeroes of the Bessel function of order zero, $J_{0}\left(x_{n}\right)=0$, with $n=1,2, \ldots$

\section{SOLUTION FOR THE CYLINDRICAL SHELL WITH STEADY CURRENT AND BATTERY}

Eqs. (13) and (14) of [5] give the solution of a conducting wire with radius $a$, length $\ell>>a$ and carrying a steady current as:

$$
\begin{gathered}
\phi(\rho \leq a, \varphi, z)=\left(\phi_{L}-\phi_{R}\right) \frac{z}{\ell}+\frac{\phi_{R}+\phi_{L}}{2} \\
\phi(\rho \geq a, \varphi, z)=\left(\phi_{L}-\phi_{R}\right) \frac{\ln (\ell / \rho)}{\ln (\ell / a)} \frac{z}{\ell}+ \\
+\frac{\phi_{R}+\phi_{L}}{2} \frac{\ln (\ell / \rho)}{\ln (\ell / a)}
\end{gathered}
$$

The solution of the problem with the battery is the sum of Eqs. (8) and (11) for the region inside the shell, and the sum of Eqs. (10) and (12) for the region outside the shell, namely:

$$
\begin{aligned}
& \phi(\rho \leq a, \varphi, z)=\frac{2 \phi_{0}}{\pi} \int_{0}^{\infty} \frac{I_{0}(k \rho)}{I_{0}(k a)} \frac{\sin (k z)}{k} d k+ \\
& +\left(\phi_{L}-\phi_{R}\right) \frac{z}{\ell}+\frac{\phi_{R}+\phi_{L}}{2} \\
& \phi(\rho \geq a, \varphi, z)=\frac{2 \phi_{0}}{\pi} \int_{0}^{\infty} \frac{K_{0}(k \rho)}{K_{0}(k a)} \frac{\sin (k z)}{k} d k+ \\
& +\left(\phi_{L}-\phi_{R}\right) \frac{\ln (\ell / \rho)}{\ln (\ell / a)} \frac{z}{\ell}+\frac{\phi_{R}+\phi_{L}}{2} \frac{\ln (\ell / \rho)}{\ln (\ell / a)}
\end{aligned}
$$

Fig. 4 shows the equipotentials with $\phi_{L}=\phi_{0}, \phi_{R}=-\phi_{0}$ and $\ell / a=10$. 


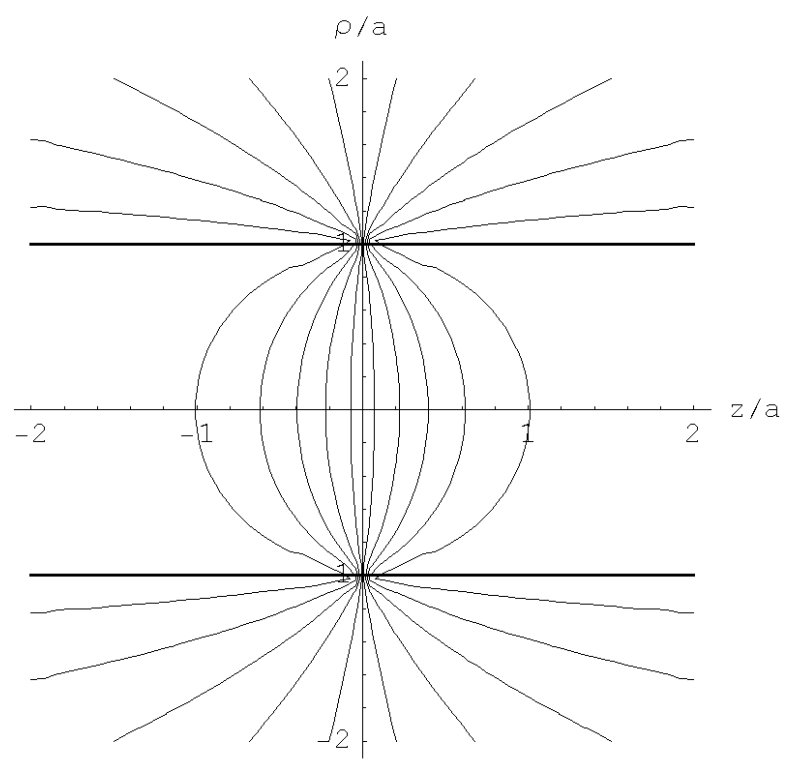

Fig. 4 Equipotentials of conducting wire close to $z=0$. This is a plot of Eqs. (13) and (14) with $\phi_{L}=-\phi_{R}=\phi_{0}$ and $\ell / a=10$. There is a steady current flowing along the positive $z$ direction.

The electric field $\vec{E}$ can be obtained from the potential utilizing the relation $\vec{E}=-\nabla \phi$. This yields:

$$
\begin{aligned}
& \vec{E}(\rho<a, \varphi, z)=-\frac{2 \phi_{0}}{\pi} \\
& {\left[\rho \int_{0}^{\infty} \frac{I_{1}(k \rho)}{I_{0}(k a)} \sin (k z) d k+z \int_{0}^{\infty} \frac{I_{0}(k \rho)}{I_{0}(k a)} \cos (k z) d k\right]+} \\
& +z \frac{\phi_{L}-\phi_{R}}{\ell} \\
& \vec{E}(\rho>a, \varphi, z)=\frac{2 \phi_{0}}{\pi} . \\
& \cdot\left[\rho \int_{0}^{\infty} \frac{K_{1}(k \rho)}{K_{0}(k a)} \sin (k z) d k-z \int_{0}^{\infty} \frac{K_{0}(k \rho)}{K_{0}(k a)} \cos (k z) d k\right]+ \\
& +\left\{\begin{array}{l}
\frac{-1}{\ln (\ell / a)} \frac{\rho}{\rho}\left[\left(\phi_{L}-\phi_{R}\right) \frac{z}{\ell}-\frac{\phi_{R}+\phi_{L}}{2}\right]+ \\
+z \frac{\phi_{L}-\phi_{R}}{\ell} \frac{\ln (\ell / \rho)}{\ln (\ell / a)}
\end{array}\right\}
\end{aligned}
$$

The surface charge distribution $\sigma(a, \varphi, z)$ can be found by applying Gauss' law and choosing a gaussian surface surrounding a small piece of the conductor surface. In the limit of an infinitesimal surface this yields the internal and external densities of surface charge as given by, respectively (where $\varepsilon_{0}=8.85 \times 10^{-12} \mathrm{C}^{2} \mathrm{~N}^{-1} \mathrm{~m}^{-2}$ ):

$$
\begin{aligned}
& \sigma_{\text {in }}\left(a^{-}, \varphi, z\right)=-\lim _{\rho \rightarrow a} \varepsilon_{0} E_{\rho}(\rho<a, \varphi, z)= \\
& =\frac{2 \phi_{0} \varepsilon_{0}}{\pi} \int_{0}^{\infty} \frac{I_{1}(k a)}{I_{0}(k a)} \sin (k z) d k= \\
& =\frac{2 \phi_{0} \varepsilon_{0}}{a} \frac{z}{|z|} \sum_{n=1}^{\infty} \mathrm{e}^{-x_{n}|z| / a} \\
& \sigma_{\text {ex }}\left(a^{+}, \varphi, z\right)=\lim _{\rho \rightarrow a} \varepsilon_{0} E_{\rho}(\rho>a, \varphi, z)= \\
& =\frac{2 \phi_{0} \varepsilon_{0}}{\pi} \int_{0}^{\infty} \frac{K_{1}(k a)}{K_{0}(k a)} \sin (k z) d k+ \\
& -\frac{\varepsilon_{0}\left(\phi_{L}-\phi_{R}\right)}{a \ell \ln (\ell / a)} z+\frac{\varepsilon_{0}\left(\phi_{R}+\phi_{L}\right)}{2 a \ln (\ell / a)}
\end{aligned}
$$

Fig. 5 shows these normalized densities of surface charge as a function of $z / a$. Both of them diverge to infinity when $|z| / a<<1$. For $|z| / a>>1$ the internal density of surface charge goes to zero faster than the external one.

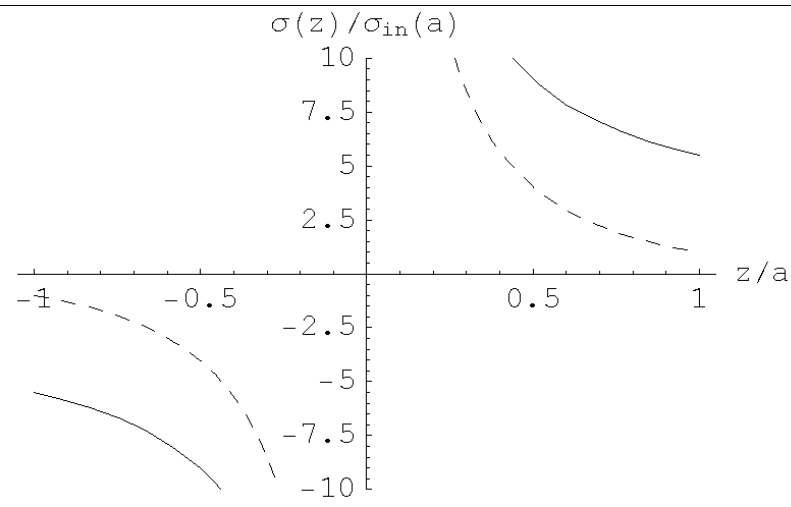

Fig. 5 Densities of surface charge as a function of the $z$ (longitudinal) coordinate for a resistive hollow cylindrical shell carrying a steady current. The dashed (continuous) lines is the normalized internal (external) density of surface charges. Both densities are normalized by the internal density $\left(\rho=a^{-}\right)$at $z=a$ 


\section{DISCUSSION}

Jefimenko and Heald considered a similar geometry (resistive cylindrical shell of radius $a$ coaxial with the $z$ axis) but carrying a steady azimuthal current, [18], [10] and [16]. A line battery located at $(\rho, \varphi, z)=(a, \pi, z)$ had its terminals at potentials $\pm \phi_{0}$. They found equal internal and external surface charge densities given by:

$$
\sigma_{\text {in }}=\sigma_{\mathrm{ex}}=\frac{\varepsilon_{0} \phi_{0}}{\pi a} \tan \frac{\varphi}{2}
$$

For angles close to the battery, $\varphi=(\pi \pm \delta) \mathrm{rad}$, with $0<\delta<<\pi$, this reduces to $\sigma_{\text {in }}=\sigma_{\text {ex }} \approx \pm 2 \varepsilon_{0} \phi_{0} / \pi a \delta$. This indicates that $\sigma$ diverges inversely proportional to the distance $d=a \delta$ to the battery.

A numerical fitting of Eq. (18) with the function:

$$
\sigma_{\text {in }}(|z|<<a)=\frac{2 \varepsilon_{0} \phi_{0} \alpha}{\pi z}
$$

for the region $|z| / a<10^{-2}$ yields the value of the unknown dimensionless constant $\alpha$ as given by $\alpha=0.998$. This indicates that $\alpha$ should be exactly equal to 1 , as expected.

We can also approximate the summation of Eq. (18) by supposing that the zeroes $x_{n}$ of the Bessel function of order zero, $J_{0}\left(x_{n}\right)=0$, are evenly spaced. That is, supposing $x_{n} \approx n x_{0}$, where $x_{0}=3.12988$ and $n=1,2, \ldots$ Using the known series:

$$
\sum_{n=1}^{\infty} x^{n}=\frac{x}{1-x}
$$

we obtain:

$$
\begin{aligned}
& \sum_{n=1}^{\infty} \mathrm{e}^{-x_{n}|z| / a} \approx \sum_{n=1}^{\infty} \mathrm{e}^{-n x_{0}|z| / a}= \\
& =\sum_{n=1}^{\infty}\left(\mathrm{e}^{-x_{0}|z| / a}\right)^{n}=\frac{1}{\mathrm{e}^{-x_{0}|z| / a}-1} \approx \frac{a}{x_{0}|z|}
\end{aligned}
$$

where in the last approximation the observation point is close to the battery, $|z| / a<<1$. Eq. (18) can be written in this approximations as:

$$
\sigma_{\text {in }}\left(a^{-}, \varphi,|z|<<a\right) \approx \frac{2 \varepsilon_{0} \phi_{0}}{x_{0} z}
$$

Eqs. (20) and (23) differ by $0.6 \%$.

A similar result can be obtained by approximating the integrals that appear in Eqs. (17) and (18) for the region $|z| / a<<1$. For Eq. (17), we divide the integral in two regions, $0<k<1 / a$ and $1 / a<k<\infty$. Defining $x \equiv k a$ and utilizing $\sin \alpha \approx \alpha$ for $\alpha<<1$ rad yields:

The functions $I_{v}(x<<1)$ and $I_{v}(x>>1)$ can be approxifhated as, [AW95, pp. 664-671]:

$$
\begin{gathered}
\int_{0}^{\infty} \frac{I_{1}(k a)}{I_{0}(k a)} \sin (k z) d k=\frac{1}{a} \int_{0}^{\infty} \frac{I_{1}(x)}{I_{0}(x)} \sin \left(\frac{x z}{a}\right) d x= \\
=\frac{1}{a} \int_{0}^{1} \frac{I_{1}(x)}{I_{0}(x)} \sin \left(\frac{x z}{a}\right) d x+\frac{1}{a} \int_{1}^{\infty} \frac{I_{1}(x)}{I_{0}(x)} \sin \left(\frac{x z}{a}\right) d x \\
\approx \frac{1}{a} \int_{0}^{1} \frac{I_{1}(x)}{I_{0}(x)} \frac{x z}{a} d x+\frac{1}{a} \int_{1}^{\infty} \frac{I_{1}(x)}{I_{0}(x)} \sin \left(\frac{x z}{a}\right) d x \\
I_{v}(x<<1) \approx\left(\frac{x}{2}\right)^{v}\left[\frac{1}{\Gamma(v+1)}+\left(\frac{x}{v}\right)^{2} \frac{1}{\Gamma(v+2)}\right], \\
I_{v}(x>>1) \approx \frac{\mathrm{e}^{x}}{\sqrt{2 \pi x}}\left(1+\frac{1-4 v^{2}}{8 x}\right)
\end{gathered}
$$

With these results we can neglect the first integral of Eq. (19) and approximate the result as:

$$
\begin{aligned}
& \int_{0}^{\infty} \frac{I_{1}(k a)}{I_{0}(k a)} \sin (k z) d k \approx \frac{1}{a} \int_{1}^{\infty} \sin \left(\frac{x z}{a}\right) d x \approx \\
& \approx \frac{1}{a} \int_{0}^{\infty} \sin \left(\frac{x z}{a}\right) d x \approx \frac{1}{z}
\end{aligned}
$$

where in the last approximation we extended the integral from 0 to $\infty$, because the integral from 0 to 1 is also negligible in the approximation $|z| / a<<1$. To arrive at the final value of $1 / z$ we utilized that the sequence $\int_{0}^{N} \sin (k x) d x=(1-\cos (k N)) / k$ diverges as $N \rightarrow \infty$, but it is weakly convergent for a suitable chosen set of test functions $g(k)$ defined for $0 \leq k<\infty$, [20].

Analogously for Eq. (18), the approximation of the integral yields: 


$$
\int_{0}^{\infty} \frac{K_{1}(k a)}{K_{0}(k a)} \sin (k z) d k \approx \frac{1}{z} .
$$

Eqs. (17) and (18) can be written in this approximations as:

$$
\begin{gathered}
\sigma_{\text {in }}\left(a^{-}, \varphi,|z|<<a\right)=\frac{2 \varepsilon_{0} \phi_{0}}{\pi z}, \\
\sigma_{\text {ex }}\left(a^{+}, \varphi, z\right)=\frac{2 \phi_{0} \varepsilon_{0}}{\pi z}-\frac{\varepsilon_{0}\left(\phi_{L}-\phi_{R}\right)}{a \ell \ln (\ell / a)} z+ \\
+\frac{\varepsilon_{0}\left(\phi_{R}+\phi_{L}\right)}{2 a \ln (\ell / a)}
\end{gathered}
$$

For a long cylindrical conductor ( $\ell>>a$ ), Eqs. (28) and (29) show that the surface charges diverge in the vicinity of the battery as $a / z$ when $z \rightarrow 0$. Additionally, in the vicinity of the battery the electrostatic contribution is greater than the terms that maintain the current flow. This implies that the situations with and without current have little difference in the surface charge distribution, although they correspond to different phenomena, as had already been observed by Jackson, [11].

The total surface charges for the symmetric case $\phi_{L}=-\phi_{R}=\phi_{0}$ can be written as:

$$
\begin{aligned}
& \sigma(a, z)=\sigma_{\text {in }}(a, z)+\sigma_{\text {ex }}(a, z)= \\
& =\frac{2 \varepsilon_{0} \phi_{0}}{\pi} \int_{0}^{\infty}\left[\frac{I_{1}(k a)}{I_{0}(k a)}+\frac{K_{1}(k a)}{K_{0}(k a)}\right] \sin (k z) d k+ \\
& -\frac{2 \varepsilon_{0} \phi_{0}}{a \ell \ln (\ell / a)} z
\end{aligned}
$$

We want to find the position $z_{0}$ where the surface charge distribution is null, $\sigma\left(a, z_{0}\right)=0$. In the interval $1 \leq \ell / a \leq 100$, equating Eq. (31) to zero yields $z_{0} / a=0.0890+0.05068 \ell / a, \quad$ or $\quad z_{0} \approx 0.507 \ell$ for $\ell / a>>1$. Although our solution is valid only for $z<<\ell / 2$ (we have neglected border effects), we found that the surface charges are null at the extremities of the cylindrical shell. This can be interpreted as follows: if we bend the wire, so that the extremities at $z= \pm \ell / 2$ touch each other, the surface charges at that position should be null. This is very reasonable.

\section{ACKNOWLEDGEMENTS}

The authors wish to thank Fapesp (Brazil) for financial support to DRCC/IFGW/Unicamp in the past few years. They thank also Regina Fonseca Avila for useful suggestion related with the integration in the complex plane. One of the authors (JAH) wishes to thank CNPq (Brazil) for financial support.

\section{APPENDIX}

Calculation of the integral of Eq. (8). As it is an even function of $k$ it can be written as:

$$
\begin{aligned}
& \int_{0}^{\infty} \frac{I_{0}(k \rho)}{I_{0}(k a)} \frac{\sin (k z)}{k} d k=\frac{1}{2} \int_{-\infty}^{\infty} \frac{I_{0}(k \rho)}{I_{0}(k a)} \frac{\sin (k z)}{k} d k= \\
& =\frac{1}{2 i} \int_{-\infty}^{\infty} \frac{I_{0}(k \rho)}{I_{0}(k a)} \frac{e^{i k z}}{k} d k
\end{aligned}
$$

The integral above is part of a contour integral on the complex variable $k$ :

$$
I=\oint_{C} \frac{I_{0}(k \rho)}{I_{0}(k a)} \frac{e^{i k z}}{k} d k,
$$

with an appropriate contour $C$. The integrand has a simple pole in $k_{0}=0$ and infinite simple poles in $k_{n}$ such that:

$$
I_{0}\left(k_{n} a\right)=0 \quad \rightarrow \quad J_{0}\left(x_{n}\right)=0,
$$

where $x_{n}=-i k_{n} a$ are the zeroes of the function $J_{0}(x)$ : $x_{1}=2.4048, \quad x_{2}=5.5201, \quad \ldots \quad$ and $n=1,2, \ldots \quad$ As $\mathrm{e}^{i k z}=\mathrm{e}^{i \alpha z-\beta z}$, for $k=\alpha+i \beta \quad(\alpha$ and $\beta$ being real numbers), the integral converges for $\beta z>0$. We choose a contour of the type shown in Fig. 6 for $z>0$. The contour integral can thus be divided in three parts: along the real $k$ axis, along the path $C_{r}$ and along the path $C_{R}$. The integral along the path $C_{r}$ is given by:

$$
\begin{aligned}
& \lim _{r \rightarrow 0} \int_{C_{r}} \frac{I_{0}(k \rho)}{I_{0}(k a)} \frac{\mathrm{e}^{i k z}}{k} d k= \\
& =\lim _{r \rightarrow 0} \int_{\pi}^{0} \frac{I_{0}\left(r \mathrm{e}^{i \varphi} \rho\right) \exp \left(i k r e^{i \varphi}\right)}{I_{0}\left(r \mathrm{e}^{i \varphi} a\right)} i r e^{i \varphi} d \varphi=-i \pi \\
& r e^{i \varphi}
\end{aligned}
$$




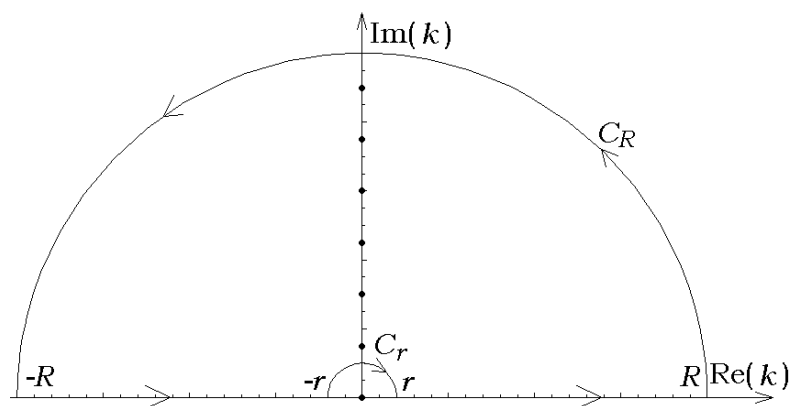

Fig. 6 Contour to calculate the integral of Eq. (8), for $z>0$. For $z<0$ we choose a symmetrical contour reflected at the horizontal (real $k$ ) axis

The integral along the path $C_{R}$ is limited, and vanishes for $R \rightarrow \infty$ :

$$
\lim _{R \rightarrow \infty}\left|\int_{C_{R}} \frac{I_{0}(k \rho)}{I_{0}(k a)} \frac{\mathrm{e}^{i k z}}{k} d k\right| \leq \lim _{R \rightarrow \infty} \int_{C_{R}}\left|\frac{\mathrm{e}^{i k z}}{k}\right| d k=0
$$
given by:

Using Cauchy' s theorem, the integral $I$ is

$$
\begin{aligned}
& \lim _{\substack{r \rightarrow 0 \\
R \rightarrow \infty}} \int_{C} \frac{I_{0}(k \rho)}{I_{0}(k a)} \frac{\mathrm{e}^{i k z}}{k} d k=\int_{-\infty}^{\infty} \frac{I_{0}(k \rho)}{I_{0}(k a)} \frac{\mathrm{e}^{i k z}}{k} d k-i \pi= \\
& =2 i \pi \sum_{n=1}^{\infty} \operatorname{Res}\left(k_{n}\right)=2 i \pi \sum_{n=1}^{\infty} \frac{I_{0}\left(k_{n} \rho\right)}{I_{1}\left(k_{n} a\right)} \frac{\mathrm{e}^{i k_{n} z}}{a k_{n}}
\end{aligned}
$$

where $\operatorname{Res}\left(k_{n}\right)$ is the residue of the integrand in $k=k_{n}=i x_{n} / a$. In the equation above we utilized the relation $I_{0}{ }^{\prime}(x)=I_{1}(x)$ involving modified Bessel functions, where $I_{0}{ }^{\prime}(x) \equiv d I_{0}(x) / d x$. Therefore the integral of Eq. (8) for $z>0$ is given by (using the relation $\left.J_{v}(i x)=i^{v} I_{v}(x)\right)$ :

$$
\int_{0}^{\infty} \frac{I_{0}(k \rho)}{I_{0}(k a)} \frac{\sin (k z)}{k} d k=\frac{\pi}{2}-\pi \sum_{n=1}^{\infty} \frac{J_{0}\left(x_{n} \rho / a\right)}{J_{1}\left(x_{n}\right)} \frac{\mathrm{e}^{-x_{n} z / a}}{x_{n}}
$$

Analogously, the integral of Eq. (8) for $z<0$ is given by:

$$
\int_{0}^{\infty} \frac{I_{0}(k \rho)}{I_{0}(k a)} \frac{\sin (k z)}{k} d k=-\frac{\pi}{2}+\pi \sum_{n=1}^{\infty} \frac{J_{0}\left(x_{n} \rho / a\right)}{J_{1}\left(x_{n}\right)} \frac{\mathrm{e}^{x_{n} z / a}}{x_{n}}
$$

\section{REFERENCES}

[1] A.K.T. Assis and J.I. Cisneros. The problem of surface charges and fields in coaxial cables and its importance for relativistic physics. Open Questions in Relativistic Physics, pp. 177-185. F. Selleri (editor), Apeiron, Montreal, pp.508-511, 1998.

[2] A.K.T. Assis and J.I. Cisneros. Surface charges and fields in a resistive coaxial cable carrying a constant current. IEEE Transactions on Circuits and Systems I, 47:63-66, 2000.

[3] A.K.T. Assis, J.A. Hernandes and J.E. Lamesa. Surface charges in conductor plates carrying constant currents. Foundations of Physics, 31:1501-1511, 2001.

[4] A.K.T. Assis and A.J. Mania. Surface charges and electric field in a two-wire resistive transmission line. Revista Brasileira de Ensino de Física, 21:469-475, 1999.

[5] A.K.T. Assis, W.A. Rodrigues Jr., and A.J. Mania. The electric field outside a stationary resistive wire carrying a constant current. Foundations of Physics, 29:729-753, 1999.

[6] G.B. Arfken and H.J. Weber. Mathematical Methods for Physicists. Academic Press, San Diego, 4th edition, Chap. 11, 1995.

[7] E. Butkov. Mathematical Physics. AddisonWesley, Reading, pp. 403, 1968.

[8] D.J. Griffiths. Introduction to Electrodynamics. Prentice Hall, New Jersey, 3rd edition, pp. 336337, 1999.

[9] J.A. Hernandes and A.K.T. Assis. The potential, electric field and surface charges for a resistive long straight strip carrying a steady current. American Journal of Physics, 71:938-942, 2003.

[10] M.A. Heald. Electric fields and charges in elementary circuits. American Journal of Physics, 52:522-526, 1984.

[11] J.D. Jackson. Surface charges on circuit wires and resistors play three roles. American Journal of Physics, 64:855-870, 1996.

[12] O.D. Jefimenko. Electricity and Magnetism. Plenum, New York, Prob. 9.33 and Fig. 14.7, 1966. 
[13] O.D. Jefimenko. Electricity and Magnetism. Electret Scientific Company, Star City, 2nd edition, pp. 318 and 509-511, 1989.

[14] A. Sommerfeld. Electrodynamics. Academic Press, New York, pp. 125-130, 1964.

[15] J.A. Stratton. Electromagnetic Theory. McGrawHill, New York, pp. 262, 1941.

[16] O.D. Jefimenko. Electricity and Magnetism. Electret Scientific Company, Star City, 2nd edition, pp. 509-511, 1989.
[17] E. Butkov. Mathematical Physics. AddisonWesley, Reading, pp. 403, 1968.

[18] O.D. Jefimenko. Electricity and Magnetism. Electret Scientific Company, Star City, 2nd edition, Prob9.33 and Fig. 14.7, 1989.

[19] E. Butkov. Mathematical Physics. AddisonWesley, Reading, pp. 312, 1968. 